\title{
Robust stabilization of the multilinked object
}

\author{
Yuliya Lezhnina ${ }^{1, *}$, Galina Ternovaya ${ }^{2}$, and Elena Evsina ${ }^{1}$ \\ ${ }^{1}$ Astrakhan State University of Architecture and Civil Engineering, CAD\&M department, 414056, str. Tatischeva, 18, Astrakhan, Russia \\ ${ }^{2}$ Astrakhan State Technical University, Department of higher and apply mathematics, 414056, str. Tatischeva, 16, Astrakhan, Russia
}

\begin{abstract}
We consider the problem of robust control for a multilink object in the presence of the signal and parametrical uncertainty when the measurement of the derivatives of input and output signals of the local subsystems is not available. Proposed and justified the use of auxiliary circuits for each of the subsystems that eliminates the use of the vector of regression during the generate of the control signal, where by the reduced order closed-loop control system. The advantage of the proposed robust control law is that it remain unchanged when exposed to unknown disturbances, and in their absence. In the first case, the system is dissipative, while in the second case, it one is asymptotically stable.
\end{abstract}

\section{Introduction}

Problem of control of uncertain objects is one of the classical problems of control theory. Complex multiply linked objects take a special place among object of control, the control of which is complicated by the linkages between local subsystems. The large number of the interconnected subsystems influencing the each other complicates the traditional control problems, and the requirement of the decentralization comes to the fore [1, 2]. The utilization of the decentralized algorithms corresponds to the nature of large interconnected systems, because it expects the distribution of the system components in space. And in addition to this, the decentralized management structure allows getting more qualitative and trusted control system, because it brings the governing body to the object and simplifies the system structure considerably $[3,4,5]$.

In this paper, we consider that class of multilinked objects, which mathematical models can be brought to a canonical form [6, 7]. To assess the perturbations introduced auxiliary circuit and the observer derivative. Compensation of the perturbations produced by the control law with opposite sign to the assessment of disturbances. The control law is constructed in such way as to offset the impact of local interconnections of subsystems $[8,9,10,11]$. There are developed a lot of methods of construct of adaptive and robust systems under parametric uncertainty of the object in the modern control theory. There are developed method extended errors, the method bypass, the algorithms of high order. At the core of these methods is the use of the filter or shunt device. A control signal is generated as the scalar product of vector of adjustable parameters and vector of regression. However, the task of creating a simple to implement and low-dimensional algorithms is still relevant. In this paper, we consider the stabilization problem for a dynamic object.

\section{Statement of the problem}

Let us examine the interconnected system with its dynamic processes which are described by the equations in the local subsystems so:

$$
\begin{aligned}
& Q_{i}(P) y_{i}(t)=R_{i}(P)\left(u_{i}(t)+f_{i}\left(y_{i}, t\right)+\sum_{i=1, i \neq j}^{k} y_{s i j}(t)\right),(1) \\
& s_{i j}=A_{s i j} s_{i j}+B_{s i j} y_{j}, \quad y_{s i j}=L_{i j} s_{i j}, \\
& i \neq j, \quad i=\overline{1, k} .
\end{aligned}
$$

where $Q_{i}(P), R_{i}(P)$ - are linear differentiation operators whose elements depend on the vector of unknown parameters $\xi \in \Xi ; \Xi-$ is a known aggregate of possible values of the vector $\xi ; \operatorname{deg} Q_{i}=n_{i}, \operatorname{deg} R_{i}=m_{i}$; $f_{i}\left(y_{i}, t\right)$ - is an unknown bounded function; $y_{i}(t)$ - is a scalar controlled variable of $i$-th subsystem which is accessible for measurement; $u_{i}(t)$ - is a scalar control action; $s_{i j} \in \mathfrak{R}^{n_{i j}}, y_{s i j} \in \mathfrak{R} ; A_{s i j} \in \mathfrak{R}^{n_{j} \times n_{j}}, B_{s i j} \in \Re^{n_{j}}-$ are unknown numerical matrixes; $L_{i j}=[1,0, \ldots, 0]-$ is a row matrix of the appropriate orders. In this case, the equation of the control object is presented in canonical form.

The equations (1) describe the dynamic processes in the local subsystems, and (2) in the cross coupling. Their transfer functions look like this as in

$$
W_{i j}(\lambda)=L_{i j}\left(\lambda I_{n_{j}}-A_{s i j}\right)^{-1} B_{s i j}=R_{s i j}(\lambda) / Q_{s i j}(\lambda)
$$

where $i \neq j$, the orders of polynomials $R_{s i j}(\lambda), Q_{s i j}(\lambda)$ are respectively equal to the following $m_{i j}, n_{i j} ; I_{n_{j}}-$ is an identity matrix of order.

\footnotetext{
* Corresponding author: lejninau@gmail.com
} 
Hypothesis.

A1. Set $\Xi$ are known.

A2. Polynomials $R_{i}(\lambda)$ are Hurwitz.

A3. The orders of Polynomials are known, the relative degree a object is known too $n_{i}-m_{i}>1$.

A4. The perturbation actions are the bounded quantity, that is $\left|f_{i}\left(y_{i}, t\right)\right| \leq C_{i}, \quad C_{i}>0$.

A5. The matrix $A_{s i j}, B_{s j}$ is unknown, but they are Polynomials $Q_{s i j}(\lambda)$ Hurwitz.

\section{Robust stabilization of the multilinked dynamic object}

We first solve the problem of the asymptotic robast stabilization when there are no external disturbances in the object (1), i.e. $f_{i}\left(y_{i}, t\right)=0$. We have to do is to design the control system for which one condition will meet

$$
\lim _{t \rightarrow \infty}\left|y_{i}(t)\right|=0,
$$

but at the same time, use of the measurable values of the other subsystems isn't allow in the local control subsystems. Decentralized robust control is implemented only if there are used only the current information about the system for construction of local control units for each subsystem. Have the equation of the control object (1), written in canonical form as

$$
Q_{i}(P) y_{i}(t)=R_{i}(P)\left(u_{i}(t)+\sum_{j=1, i \neq j}^{k} y_{s i j}(t)\right)
$$

where $Q_{i}(P), R_{i}(P)$ - is a linear differentiation operator with a constant rates. In the generation of control law will take estimate of the output signal with opposite sign. So it form is

$$
u_{i}(t)=-\theta_{i} T_{i}(P) \bar{y}_{i}(t)
$$

where a number $\theta_{i}>0$ and a linear differentiation operator $T_{i}(P)$ of order $n_{i}-m_{i}-1$ are chosen, according to the Hurwitz of polynomial

$$
Q_{0 i}(\lambda)=Q_{i}(\lambda)+\theta_{i} R_{i}(\lambda) T_{i}(\lambda)
$$

the function $\bar{y}_{i}(t)$ is an estimate of a signal $y_{i}(t)$ which is realized by the observer [12]

$$
\dot{x}_{i}=F_{0 i} \bar{x}_{i}+H_{i}\left(y_{i}-\bar{y}_{i}\right), \quad \bar{y}_{i}=L_{0 i} \bar{x}_{i} .
$$

Here $\quad \bar{x}_{i} \in \Re^{n_{i}-m_{i}} ; L_{0 i}=[1,0, \ldots, 0] ; \quad \chi_{i}>0$ - is the little number, $F_{0 i}=\left[\begin{array}{cc}0 & I_{n_{i}-m_{i}-1} \\ 0 & 0\end{array}\right], H_{i}^{T}=\left[\frac{h_{1 i}}{\chi_{i}}, \ldots, \frac{h_{n_{i}-m_{i}-1}}{\chi_{i}^{n_{i}-m_{i}-1}}\right] ;$; the vector $H_{i}$ is chosen by special method so that the matrix $F_{i}=F_{0 i}+\bar{H}_{i} L_{0 i}$ was the Hurwitz, where $\bar{H}_{i}^{T}=\left[-h_{1 i}, \ldots,-h_{n_{i}-m_{i}-1}\right] ; I_{n_{i}-m_{i}-1}-$ is an identity matrix of order $\left(n_{i}-m_{i}-1\right) \times\left(n_{i}-m_{i}-1\right)$. It is obvious, that the control law can be technically realizable, as it contains the known or measurable values. At the time, the solution of the problem gives the following statement. The Statement 1 If the hypotheses A.1 - A.5 are implemented, then the control law (5) with the observer (7) implements of the target condition (5).

Proof of Proposition 1. Inputting the control (5) to the equation of object (1) with (6), we'll get the last input-output model in the matrix vectorial form

$$
\dot{x}_{i}=A_{0 i} x_{i}+\bar{B}_{i}\left(y_{i}-\bar{y}_{i}\right)+B_{i} \sum_{j=1, i \neq j}^{k} y_{s i j}, \quad y_{i}=L_{i} x_{i},
$$

where $\bar{B}_{i} \in \Re^{n_{i}}$ - is a vector whose components are the coefficients $\theta_{i} R_{i}(P) T_{i}(P) ; A_{0 i}$ - is a Hurwitz matrix in the form of a characteristic polynomial of the Frobenius $Q_{0 i}(\lambda) ; B_{i}^{T}=\left[0, \ldots, 0, b_{0}, \ldots, b_{m}\right]$. Following the [13], we introduce into consideration nullity vector $\eta_{i}$ as

$$
\dot{\eta}_{i}=\frac{1}{\chi_{i}} F_{i} \eta_{i}+B_{1 i} x_{21 i}+D_{i}^{-1} B_{1 i} L_{3 i} \eta_{i}
$$

where $D_{i}=\operatorname{diag}\left\{\chi_{i}^{n_{i}-m_{i}-1}, \ldots, \chi_{i}, 1\right\}, x_{1 i}$ - is a component part of the state vector, namely $x_{i}^{T}=\left[x_{1 i}^{T}, x_{2 i}^{T}\right]$.

We introduce the augmented state vector $z_{i}=\operatorname{col}\left(x_{i}, \eta_{i}\right)$ and write the equation (8), (9) in the composite matrix vectorial form

$$
\begin{aligned}
z_{i} & =B_{1 p i} x_{21 i}+\bar{D}_{i} B_{3 p i} L_{3 i} \eta_{i}+ \\
& +\frac{1}{\chi_{i}} A_{p i} z_{i}+B_{2 p i} \sum_{j=1}^{k} y_{s i j} \\
y_{p i} & =L_{p i} z_{i},
\end{aligned}
$$

and at the same time matrixes $A_{p i}$ will be Hurwitz.

Let us take the Lyapunov function in the form of

$$
V=\sum_{i=1}^{k}\left\{z_{i}^{T}(t) P_{i} z_{i}(t)+\sum_{j=1}^{k} s_{i j}^{T} P_{s i j} s_{i j}\right\}
$$

in which $P_{i}, P_{s i j}$ - are the positive definite symmetric matrixes satisfy the matrix relation:

$$
\begin{aligned}
& P_{i} A_{p i}+A_{p i}^{T} P_{i}=-Q_{p i}, \\
& Q_{p i}=Q_{i}+\left(\rho_{1 i}+\rho_{2 i}\right) I_{2 n_{i}-m_{i}-1}, \\
& P_{s i j} A_{s i j}+A_{s i j}^{T} P_{s i j}=-Q_{p i j}, \\
& Q_{p i j}=Q_{s i j}+\rho_{s i j} I_{n_{i j}} .
\end{aligned}
$$

Because the matrixes $A_{p i}$ and $A_{s i j}$ are Hurwitz, there are matrixes $P_{i}$ and $P_{s i j}$, that satisfy the matrix relation (12). Let us calculate the total derivative of the function (12) on the trajectories of the system (10), (2), with (12). Then the derivative of the Lyapunov function will look like 


$$
\dot{V} \leq \sum_{i=1}^{k}\left\{-\alpha_{1} V-\frac{\rho_{1 i}}{2 \chi_{i}}\left\|x_{i}\right\|^{2}\right\} \leq-\alpha_{1} V,
$$

the synthesized system is stable, because the target condition is satisfied (4).

\section{Robust stabilization of the multilinked dynamic object under bounded disturbances}

However, in practice it is difficult to create the situation for control of technical objects in ideal conditions when it is not exposed to external disturbances. The case where external disturbances are bounded is the simplest. Next, let us solve the problem of a decentralised stabilization when the external disturbances are present in the object (1), i.e. $f_{i}\left(y_{i}, t\right) \neq 0$. We had to design a control system for which next condition will be satisfied

$$
\varlimsup_{t \rightarrow \infty}\left|y_{i}(t)\right|<\delta,
$$

In accordance with the approach presented in $[13,14]$ let us ask one local control law in the form of (5) where a number $\theta_{i}>0$ and the linear differentiation operator $T_{i}(P)$ of order $n_{i}-m_{i}-1$ are chosen from the reasons of Hurwitz polynomial (6), and the function $\bar{y}_{i}(t)$ is an estimate of output $y_{i}(t)$. Then the equation (1) will look like

$$
\begin{aligned}
Q_{0 i}(P) y_{i}(t) & =R_{i}(P)\left(\theta_{i} T_{i}(P)\left(y_{i}(t)-\bar{y}_{i}(t)\right)+\right. \\
& \left.+f_{i}\left(y_{i}, t\right)+\sum_{j=1, i \neq j}^{k} y_{s i j}(t)\right)
\end{aligned}
$$

Implementing the control law (5) requires getting the estimate $\bar{y}_{i}(t)$ and its $n_{i}-m_{i}-1$ derivatives, for which we will use the observer (7). It is obvious, that the control law can be technically realizable, as it contains the known or measurable values.

The Statement 2. If the hypotheses A.1 - A.5 are implemented, then the control law (5) with the observer (7) implements the limited nature of system trajectory (14).

Observation. But it should be noted, that choosing the number $\theta_{i}$ of greater value, and the value $\chi$ of smaller, we can achieve the target condition (14).

Proof of Proposition 2. Let us convert the equation (14) to the vectorial matrix form

$$
\begin{aligned}
& \dot{x}_{i}(t)=A_{0 i} x_{i}(t)+ \\
& +B_{i}\left(\tau_{i}^{T}\left(x_{1 i}(t)-\bar{x}_{i}(t)\right)+f_{i}\left(y_{i}, t\right)+\sum_{j=1, i \neq j}^{k} y_{s i j}(t)\right),(15) \\
& y_{i}(t)=L_{i} x_{i}(t),
\end{aligned}
$$

where $x_{i} \in \Re^{n_{i}} ; y_{i} \in \mathfrak{R} ; A_{0 i}-$ is a Hurwitz matrix in Frobenius form with characteristic polynomial $Q_{0 i}(\lambda)$; $\tau_{i} \in \Re^{n_{i}-m_{i}}-$ is a vector whose components are the coefficients $\quad \theta_{i} T_{i}(P) ; \quad B_{i}^{T}=\left[0, \ldots, 0, b_{0 i}, \ldots, b_{m i}\right]$; $L_{i}=[1,0, \ldots, 0] ; x_{1 i}-$ is a component part of the state vector, namely $x_{i}^{T}=\left[x_{1 i}^{T}, x_{2 i}^{T}\right]$ :

$$
\begin{aligned}
& \dot{x}_{1 i}(t)=F_{0 i} x_{1 i}(t)+B_{0 i}\left(\frac{1}{b_{0 i}} x_{21 i}+\right. \\
& \quad+\tau_{i}^{T}\left(x_{1 i}(t)-\bar{x}_{i}(t)\right)+f_{i}\left(y_{i}, t\right)+\sum_{j=1, i \neq j}^{k} y_{s i j}(t), \\
& \dot{x}_{2 i}(t)=F_{1 i} x_{2 i}(t)+B_{1 i} c_{1 i}^{T} x_{i}(t)+ \\
& +B_{1 i}\left(\tau_{i}^{T}\left(x_{1 i}(t)-x_{i}(t)\right)+f_{i}\left(y_{i}, t\right)+\sum_{j=1, i \neq j}^{k} y_{s i j}(t)\right), \\
& y_{i}(t)=L_{0 i} x_{1 i}(t),
\end{aligned}
$$

where the matrix $F_{1 i}$ has the same structure as $F_{0 i}$; $B_{0 i}^{T}=\left[0, \ldots, 0, b_{0 i}\right] ; \quad x_{1 i} \in \mathfrak{R}^{n_{i}-m_{i}} ; x_{2 i} \in \mathfrak{R}^{m_{i}}, \quad$ the vector components $c_{1 i}$ are the coefficients $Q_{0 i}(\lambda)$ with opposite signs; $B_{1 i}^{T}=\left[b_{1 i}, \ldots, b_{m i}\right] ; x_{21 i}-$ is the first component of vector $x_{2 i} ; L_{0 i}=[1,0, \ldots, 0]$. Following the [13], we introduce the vector (9). Then from (7) and (16) we have

$$
\begin{aligned}
& \dot{\eta}_{i}(t)=\frac{1}{\chi} F_{i} \eta_{i}(t)+B_{0 i}\left(\tau_{i}^{T} D_{i} \eta_{i}(t)+\frac{1}{b_{0 i}} x_{21 i}+\right. \\
& =f_{i}\left(y_{i}, t\right)+\sum_{j=1, i \neq j}^{k} y_{s i j}(t)
\end{aligned}
$$

Structure of matrix $F_{0 i}, B_{1 i}, L_{0 i}, D_{i}, H_{i}$ is such that the equalities are satisfied

$$
D_{i}^{-1}\left(F_{0 i}-H_{i} L_{i}\right) D_{i}=\frac{1}{\chi} F_{i}, \quad D_{i}^{-1} B_{0 i}=B_{0 i},
$$

Let us introduce block diagonal matrix

$F=\operatorname{diag}\left\{F_{1}, \ldots, F_{k}\right\} ; \quad B_{0}=\operatorname{diag}\left\{B_{01}, \ldots, B_{0 k}\right\}$,

$D=\operatorname{diag}\left\{D_{1}, \ldots, D_{k}\right\} ; \bar{B}_{0}=\operatorname{diag}\left\{b_{01}^{-1}, \ldots, b_{0 k}^{-1}\right\}$,

$A_{0}=\operatorname{diag}\left\{A_{01}, \ldots, A_{0 k}\right\} ; \quad B=\operatorname{diag}\left\{B_{1}, \ldots, B_{k}\right\}$,

and the vectors $\eta=\operatorname{col}\left(\eta_{1}, \ldots, \eta_{k}\right) ; \tau=\operatorname{col}\left(\tau_{1}, \ldots, \tau_{k}\right)$,

$x=\operatorname{col}\left(x_{1}, \ldots, x_{k}\right) ; x_{21}=\operatorname{col}\left(x_{211}, \ldots, x_{21 k}\right)$;

$f=\operatorname{col}\left(f_{1}, \ldots, f_{k}\right)$;

$y_{s i}=\operatorname{col}\left(y_{s 11}, \ldots, y_{s i k}\right) ; y_{s}=\operatorname{col}\left(y_{s 1}, \ldots, y_{s k}\right)$,

Then the equation (15), (17) in the composite form will look like

$$
\begin{aligned}
\dot{\eta}(t) & =\frac{1}{\chi} F \eta(t)+B_{0}\left(\tau^{T} D \eta+B_{0} x_{21}+f(t)+a_{s} y_{s}\right), \\
\dot{x}(t) & =A_{0} x(t)+B\left(\tau^{T} D \eta+f(t)+a_{s} y_{s}(t)\right),
\end{aligned}
$$

where $\eta(t) \in \mathfrak{R}^{n-m} ; x(t) \in \mathfrak{R}^{n} ; a_{s}$ - is a block matrix with the blocks $I_{k}, I_{k}-$ is a identity matrix of order $k \times k$ : 


$$
a_{s}=\left[\begin{array}{cccc}
I_{k} & 0 & \cdots & 0 \\
0 & I_{k} & \cdots & 0 \\
\vdots & \vdots & \ddots & \vdots \\
0 & 0 & \cdots & I_{k}
\end{array}\right] .
$$

Let us take the Lyapunov function

$$
V_{1}=\eta^{T}(t) P \eta(t)+\sum_{i=1}^{k} \sum_{j=1, i \neq j}^{k} s_{i j}^{T} M_{s i j} s_{i j},
$$

where $P, M_{s i j}$ - are the positive definite symmetric matrixes, at the same time $P=\operatorname{diag}\left\{P_{1}, \ldots, P_{n-m}\right\}$. To take account the fact that the matrixes are block diagonal, we will produce that positive definite symmetric matrixes $P_{i}$ must satisfy the equations for every subsystem

$$
\begin{aligned}
& P_{i} F_{i}+F_{i}^{T} P_{i}=-Q_{i}-\rho_{i} I_{n_{i}-m_{i}}, \\
& M_{s i j} A_{s i j}+A_{s i j}{ }^{T} M_{s i j}=-Q_{s i j}-\rho_{s i j} I_{n_{j}},
\end{aligned}
$$

where $\quad \rho_{i}>0 ; \rho_{s i j}>0 ; \quad Q_{i}=Q_{i}{ }^{T}>0 ; Q_{s i j}=Q_{s i j}{ }^{T}>0$. Since the matrixes $F_{i}$ and $A_{s i j}$ are Hurwitz we see that there are the matrixes $P_{i}$ and $M_{s i j}$, with the ratio (19).

Let us take the Lyapunov function

$$
V_{2}=x^{T}(t) H x(t),
$$

where $H-$ are the positive definite symmetric matrixes, at the same time $H=\operatorname{diag}\left\{H_{1}, \ldots, H_{n}\right\}$. To take account the fact that the matrix $H$ is a block diagonal, we will produce that positive definite symmetric matrixes $H_{i}$ must satisfy the equations for every subsystem

$$
H_{i} A_{0 i}+A_{0 i}{ }^{T} H_{i}=-Q_{1 i}-\rho_{1 i} I_{n_{i}},
$$

where $\rho_{1 i}>0 ; Q_{1 i}=Q_{1 i}{ }^{T}>0$. Since the matrixes $A_{0 i}$ are Hurwitz we see that there are the matrixes $H_{i}$ which satisfy these matrix relation. To take account the estimated derivative of the functions (19), used (20) and the last inequality, we will calculate the total derivative of the Lyapunov function $V=V_{1}+V_{2}$, and we'll get the inequality

$$
V\left(x_{i}(t), \eta(t)\right) \leq-\beta_{3} V+\beta_{4},
$$

where

$$
\beta_{4}=\sum_{i=1}^{k} \beta_{2 i} \sup _{t} C_{i}^{2},
$$

$\beta_{3}=\min \left\{0.25 \alpha_{i} ; \frac{1}{\chi} \frac{\lambda_{\min }\left(Q_{i}\right)}{\lambda_{\max }\left(P_{i}\right)} ; \frac{\lambda_{\text {min }}\left(Q_{s i j}\right)}{\lambda_{\text {max }}\left(M_{s i j}\right)}\right\}$, from which the dissipative system follows $V \leq \frac{\beta_{4}}{\beta_{3}}$ and the estimate $\varlimsup_{t \rightarrow \infty}\left|x_{i}\right| \leq \frac{\beta_{4}}{\beta_{3}}$.

This value can be reduced by choosing a little number $\chi$ which leads to an increase of the $1 / \chi$, and $\theta_{i}$ in the (5) greater. Unfortunately, we cannot get a more accurate estimate.

\section{Example}

Consider the multilinked control plant, dynamic processes which are described by the equations

$$
\begin{aligned}
& \left(P^{3}+a_{1} P^{2}+a_{2} P+a_{3}\right) y_{1}(t)= \\
& =\left(b_{1} P+b_{2}\right) u_{1}(t)+s_{1} y_{2}(t) \\
& \left(P^{3}+a_{4} P^{2}+a_{5} P+a_{6}\right) y_{2}(t)= \\
& =\left(b_{3} P+b_{4}\right) u_{2}(t)+s_{2} y_{1}(t)
\end{aligned}
$$

Aggregate of possible values of the vector $\Xi=\left\{a_{l}, s_{l}, b, l=\overline{1,6}: a_{l} \in[-8 ; 5], b \in[1 ; 8]\right\}$.

The equation of the auxiliary circuit has the form $\left(P^{2}+2 P+1\right) \widetilde{y}_{i}=u_{i}, \quad i=1,2$.

Let's used observer

$$
\begin{aligned}
& \bar{\zeta}_{i}=\left[\begin{array}{ll}
0 & 1 \\
0 & 0
\end{array}\right] \bar{\zeta}_{i}+\left[\begin{array}{c}
3 / \mu \\
3 / \mu^{2}
\end{array}\right]\left(\bar{\vartheta}_{i}(t)-\vartheta_{i}\right), \quad, i=1,2 . \\
& \bar{\vartheta}_{i}=\left[\begin{array}{ll}
1 & 0
\end{array}\right] \bar{\zeta}_{i}
\end{aligned}
$$

The control law is in the form of

$$
u_{i}(t)=-\frac{\alpha}{k_{m}}\left(\zeta_{1 i}(t)+2 \dot{\zeta}_{1 i}(t)+\dot{\zeta}_{2 i}(t)\right), i=1,2 .
$$

General block diagram of the control object shown in fig. 1. Here we have the multilinked control object consisting of $K$ local subsystems. Control signal and the output signals from all the other $K-1$ local subsystems are inputs of each local subsystem. The local control unit generates a control signal, using an estimate of the state vector of the object that is obtained with the observer. Thus is implemented a decentralized control.

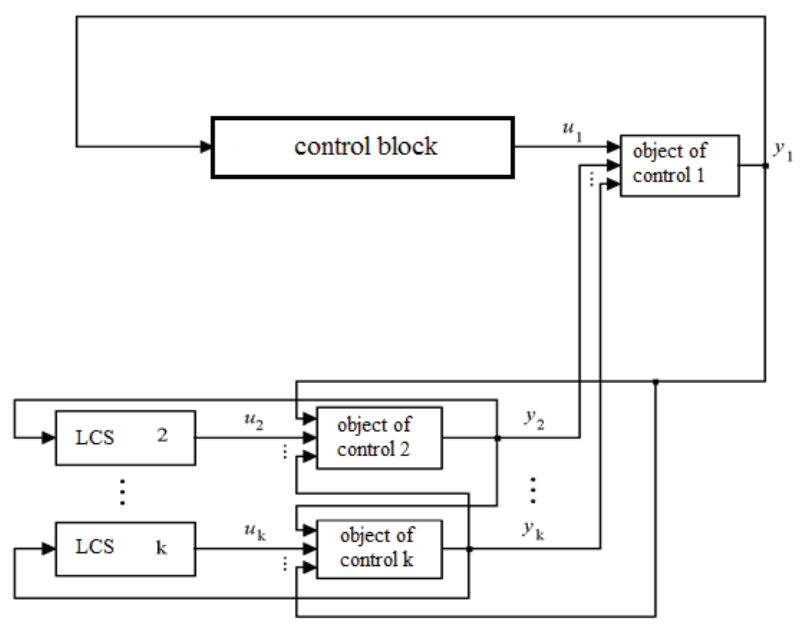

Fig.1. Structural scheme of stabilisation of the i-th subsystem

This object is synthesized with a decentralized robust controller for stabilization of each of the local subsystems, the structure of which is shown in Fig.2. The control signal is generated with a opposite sign to 
the assessment of variables of the internal state. Compensation of external and structural disturbances is possible because introducing the auxiliary circuit. The disadvantage of the proposed control law is that the absolute magnitude of the coefficients $K$ and $\alpha$ is impossible to find analytically. Their value is chosen at the modeling stage.

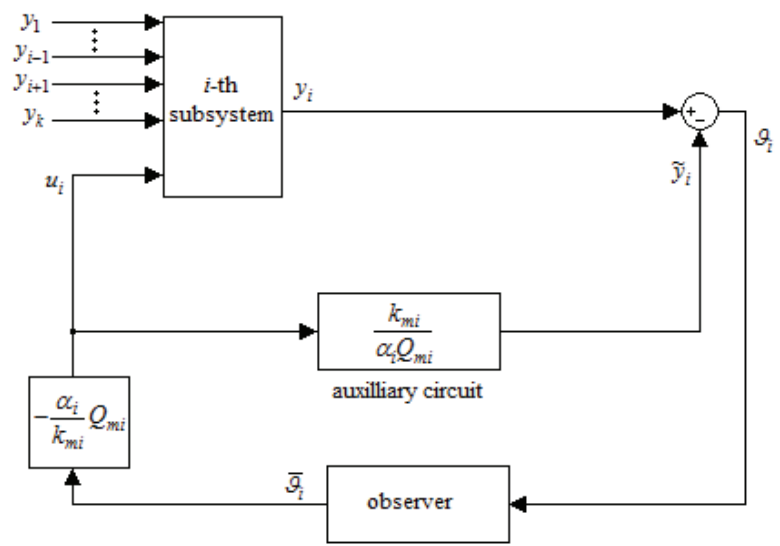

Fig.2. Structural scheme of robast control of the $i$-th subsystem

Fig. 3 shows the simulation results of the stabilization system of the unstable object in the presence of external disturbances

$$
\begin{aligned}
& f_{1}(t) \neq 0, \quad f_{2} \neq 0, \\
& y_{1}(0)=\dot{y}_{1}(0)=\ddot{y}_{1}(0)=2, \\
& y_{2}(0)=\dot{y}_{2}(0)=y_{2}(0)=-1
\end{aligned}
$$

There are follows external disturbances in the object

$f_{1}(t)=1+\sin 0,1 t+\sin 10 t$,

$f_{2}(t)=1+2 \sin 0,3 t+\sin 10 t$.

Consider the control object, dynamic processes in which case are described by the equation

$$
\begin{aligned}
& \left(P^{3}+a_{1} P^{2}+a_{2} P+a_{3}\right) y_{1}(t)=b_{1}\left(u_{1}(t)+f_{1}(y, t)+y_{s 12}(t)\right), \\
& y_{s 12}=3(p+2) y_{2}, \\
& \left(P^{3}+a_{4} P^{2}+a_{5} P+a_{6}\right) y_{1}(t)=b_{2}\left(u_{2}(t)+f_{2}(y, t)+y_{s 21}(t)\right), \\
& y_{s 21}=5 / p^{2}+p+1 y_{1} .
\end{aligned}
$$

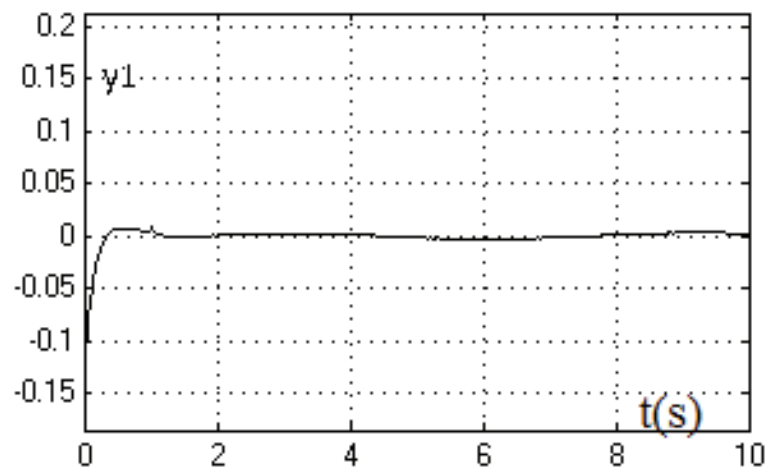

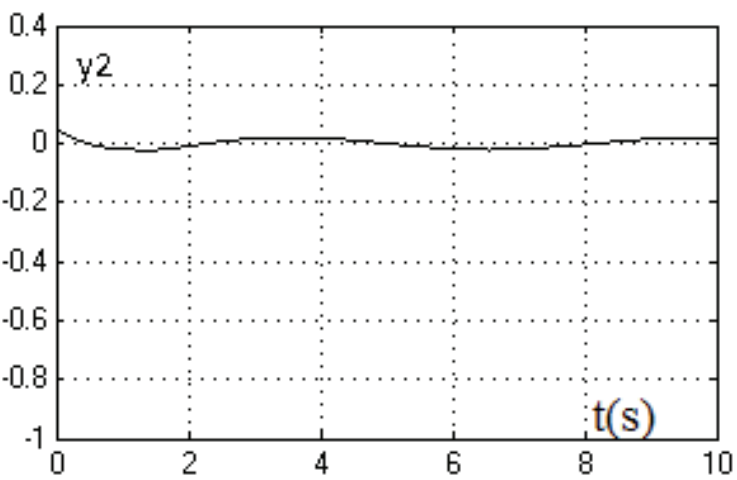

Fig.3. The trajectories of the system's outputs in robust stabilization

Aggregate of possible values of the vector $\Xi=\left\{a_{l}, s_{l}, b, l=\overline{1,6}: a_{l} \in[-5 ; 5], b \in[1 ; 5]\right\}$.

External disturbances are present as $f_{1}(t)=\sin 2 t, f_{2}(t)=2 \sin 3 t$.

We define the control law in the form (5), where $T_{i}(P)=\tau_{1 i} P^{2}+\tau_{2 i} P+\tau_{3 i}$.

Get $\theta_{i}=20, \tau_{1 i}=16, \tau_{2 i}=8, \tau_{3 i}=2$.

In this case, the polynomials $Q_{0 i}(\lambda),(i=1,2)$ are Hurwitz. To obtain the observer (7) will take the value $\chi=0.01$, and vector $H_{i}^{T}=[-3 ;-3 ;-1], \quad i=1,2$.

Fig. 4 shows a model diagram of the first subsystem of the object (1) dissipative stabilization.

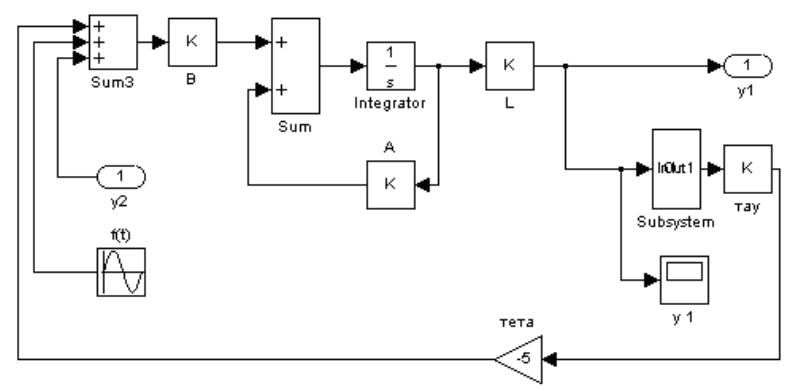

Fig.4.Structural scheme of robast control of the $i$-th subsystem.

Fig. 5 shows the simulation results of dissipative system of stabilization with perturbations $f_{1}(t)=\sin 2 t, f_{2}(t)=2 \sin 3 t$.

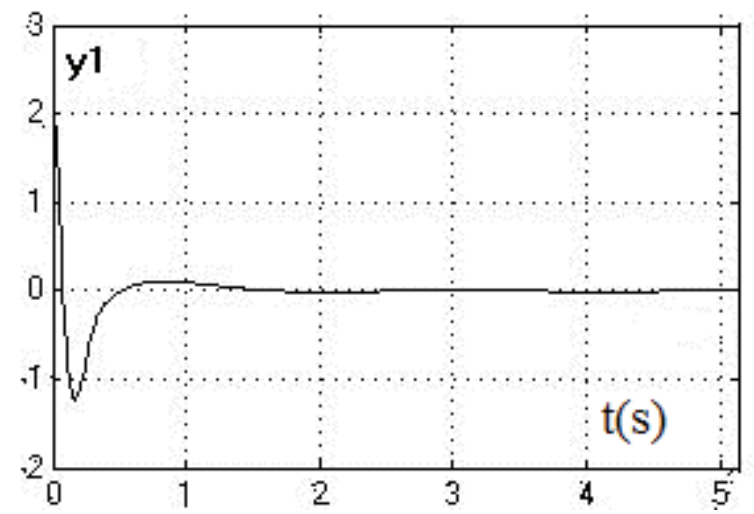




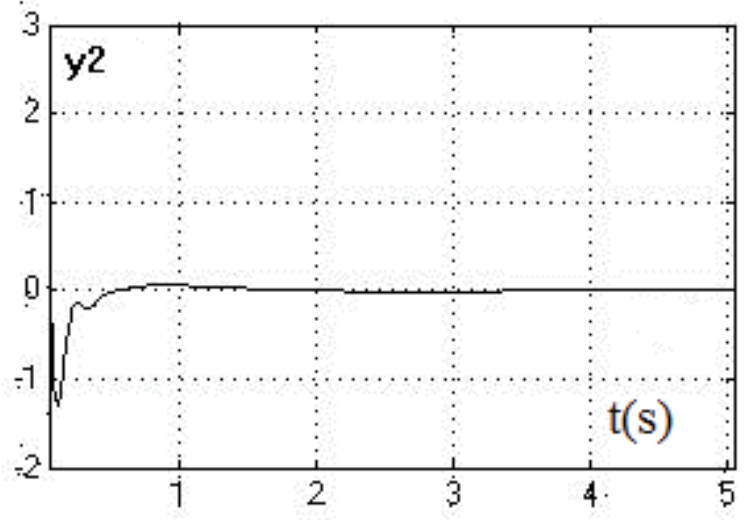

Fig. 5. The trajectories of the system's outputs in robust stabilization.

\section{Discusion}

The advantage of the proposed robust control law (5) is that it remains the same in the presence of unknown perturbation actions and in their absence. Only in the first case the system will be dissipative, while the second case it will be asymptotically robust.

\section{References}

1. Y.A. Lezhnina, G.N. Ternovaya, E.R. Galyauv, I.Y. Kvyatkovskaya, Decentralized robust control over robot manipulator, Applied Mechanics and Materials, 437 (2013)

2. I.B. Furtat, J.V. Chugina, A.L. Fradkov, Robust control of multi-machine power systems caused by perturbation of mechanical input power and variable unknown communication time-delay, IFAC-PapersOnLine 49(14) (2016)

3. A. M. Tsykunov, Robust control algorithms with compensation of bounded perturbations, Automation and Remote Control, 7 (2007)

4. Yu. Lezhnina, I. Petrova, G. Ternovay, Path Control for a Manipulator While Tracking a Given Trajectory, Applied Mechanics and Materials, pp. 752-753 (2015)

5. Yu. Lezhnina, G. Ternovaya, V. Zaripova, Robust Adaptive Control of the Dynamic Multilinked Object: Control of Robot Manipulator, Progress in Systems Engineering Proceedings of the TwentyThird International Conference on Systems Engineering (ICSEng 2014, Las Vegas, Nevada, August 19, 2014), (2014)

6. A. M. Tsykunov, Robust output control of linear dynamic objects, Mekhatronika, avtomatizatsiya, upravlenie 8 (2008)

7. E. A. Parsheva, Yu. A. Lezhnina, Robust decentralized control with disturbance compensation with nonlinear multivariable objects, Mekhatronica, avtomatizatsiya, upravlenie 8 (2011)

8. E. A.Parsheva, Robust Decentralized Control with Scalar Output of Multivariable Plants with
Uncertain Structures, 6th IFAC Symposium on Robust Control Design, 6(1) (2009)

9. V.O. Nikiforov, Robust high-order tuner of simplified structure, Automatica, 8(35) (1999)

10. A. A. Bobtsov, Robust control algorithm of uncertain object without measuring derivatives of adjusted variable, Autom. Telemekh 8 (2003)

11. I. B. Furtat, A. L.Fradkov, A. M. Tsykunov, Robust Synchronization of Linear Networks with Compensation of Disturbances, Proceedings of 18th IFAC World Congress 18(1)

12. A. N. Atassi, H. H. Khalil, A separation principle for the stabilization of a class of nonlinear systems, IEEE Trans. on Automatic Control 9(44) (1999)

13. G. Tao, P. A. Ioannou, Model reference adaptive control for plants with unknown relative degree, IEEE Trans. Automat. Control 6(38) (1993)

14. V. A. Brusin, A class of singular distributed adaptive systems, Autom. Telemekh. 4 (1995) 